

\title{
Emulation-based stabilization of networked control systems implemented on FlexRay
}

Wei Wang, Dragan Nesic, Romain Postoyan

\section{To cite this version:}

Wei Wang, Dragan Nesic, Romain Postoyan. Emulation-based stabilization of networked control systems implemented on FlexRay. Automatica, 2015, 59, pp.73-83. 10.1016/j.automatica.2015.06.010 . hal-01166719

\section{HAL Id: hal-01166719 \\ https://hal.science/hal-01166719}

Submitted on 24 Jun 2015

HAL is a multi-disciplinary open access archive for the deposit and dissemination of scientific research documents, whether they are published or not. The documents may come from teaching and research institutions in France or abroad, or from public or private research centers.
L'archive ouverte pluridisciplinaire HAL, est destinée au dépôt et à la diffusion de documents scientifiques de niveau recherche, publiés ou non, émanant des établissements d'enseignement et de recherche français ou étrangers, des laboratoires publics ou privés. 


\title{
Emulation-based stabilization of networked control systems implemented on FlexRay *
}

\author{
Wei Wang ${ }^{a}$, Dragan Nešićc ${ }^{\mathrm{a}}$, Romain Postoyan ${ }^{\mathrm{b}}$ \\ ${ }^{a}$ Department of Electrical and Electronic Engineering, the University of Melbourne, Parkville, 3010, Victoria, Australia \\ ${ }^{\mathrm{b}}$ Université de Lorraine, CRAN, UMR 7039 and CNRS, CRAN, UMR 7039, France
}

\begin{abstract}
We investigate the emulation controller design approach for nonlinear networked control systems (NCS) with FlexRay. FlexRay is a deterministic communication protocol which is increasingly used in the automotive industry as it provides a high bandwidth and allows for safety critical applications. It is characterized by pre-set communication cycles that are subdivided into static and dynamic segments; the data transmissions are scheduled by different rules depending on the segment. We propose for the first time a hybrid model of NCS with FlexRay for this purpose. We show, under reasonable assumptions, that the asymptotic stability property ensured by the controller in the absence of communication constraints is preserved when the latter is implemented over FlexRay with sufficiently frequent data transmission. In particular, we assume that on each communication segment, the data transmissions are governed by uniformly globally exponentially stable protocols. This covers the case when the round-robin protocol is implemented on the static segment and the try-once-discard protocol is implemented on the dynamic segment. We provide explicit maximum allowable transmission interval bounds that guarantee stability.
\end{abstract}

\section{Introduction}

Networked control systems (NCS) is a new generation of control systems in which the controller and the plant communicate via a digital network. The network is typically modeled as a serial communication channel with a finite bandwidth. NCS attract a lot of attention due to the benefits they offer in terms of reduced cost, weight and volume and ease of maintenance and installation. On the other hand, the communication channel induces undesirable constraints like time-varying sampling, time delays, scheduling, quantization and packet dropouts, which complicate the analysis and the design and in general adversely affect the behavior of NCS. In the present paper, we concentrate on the effect of time-varying data sampling and scheduling. Many solutions have been proposed to handle these constraints for NCS, see [7] for a good summary. However, it is not clear whether these

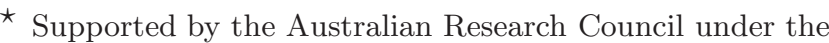
Discovery Project and Future Fellow program, the ANR under the grant COMPACS (ANR-13- BS03-0004-02) and the European 7th Framework Network of Excellence "Highlycomplex and networked control systems" (HYCON2 No. 257462).

Email addresses: wei.wang@unimelb.edu.au (Wei Wang), dnesic@unimelb.edu.au (Dragan Nešić), romain.postoyan @univ-lorraine.fr (Romain Postoyan).
}

theoretical results can be directly applied to NCS with some specific communication networks. This paper aims to bridge this gap between theory and practice by considering NCS with Flexray. There are works that deal with feedback control systems implemented on FlexRay, see $[5,12]$ for instance. We generalise these results by considering nonlinear systems (as opposed to linear) and we propose a novel model of NCS with FlexRay, which we believe would be very useful in future studies of this important class of systems.

FlexRay was developed by BMW, Daimler-Chrysler, Philips and Freescale in 2000 [3,18] to provide appropriate communications for implementing $\mathrm{x}$-by-wire technology in automotive control. FlexRay network sends data packets in pre-set communication cycles that consist of a static segment and a dynamic segment that are periodically repeated [3]. During the static segment, the network capacity is assigned to nodes in a prefixed manner: we say that the scheduling rule is static. The dynamic segment enables messages to be sent whenever it is required which helps meet varying bandwidth requirements that can emerge at system run time. A dynamic policy schedules transmissions of the nodes in this case based on the online information.

Our first contribution is a novel hybrid model for NCS with FlexRay based on the formalism of [4] that we 
derive under some simplifying but reasonable assumptions. Our goal is to provide a high fidelity model that is amenable to controller design and stability analysis; the main challenge is to model the data transmissions on the one hand and the switches between the static and the dynamic segments on the other hand. To achieve this, we first describe in detail the Flexray communication cycles and then introduce two clock variables in our model which respectively represent the time elapsed since the last data transmission and the time elapsed since the last segment switch.

Our second contribution is an emulation result for NCS with FlexRay. The main idea in emulation is to first design a controller that stabilizes the plant in the absence of the network; at this step, one can use any nonlinear continuous-time design technique to construct the controller. Then, in the second step, the controller is implemented over the network and it is shown that the stability of the system is preserved for sufficiently high communication bandwidth, which is measured in terms of the so-called maximal allowable transmission interval (MATI). This approach is applicable to a wide class of nonlinear NCS and network protocols, see $[1,7,14,21,23]$ and the references therein. However, none of the available results in the literature are directly applicable to the model of NCS with FlexRay that we propose; this is mainly due to the complexity of the FlexRay communication cycle that requires more complicated models not considered in prior works. Hence, we develop novel emulation results that are applicable in this case. In particular, we assume that, for instance, Round-Robin (RR) protocol is implemented during the static segment and Try-Once-Discard (TOD) protocol is implemented during the dynamic segment of the FlexRay communication cycle. We show that asymptotic stability of the system in the absence of network is maintained when the controller is implemented over FlexRay provided that the MATI of each segment satisfies a given bound.

The analysis relies on an original hybrid Lyapunov function construction which generalizes the one proposed in [1]. Moreover, we derive segment-dependent MATI bounds which seems to be more adequate in practice. We believe that the results of this paper, in particular the hybrid model and the constructed Lyapunov function, could be used as a starting point to investigate other problems such as estimation or tracking control for NCS over FlexRay. A preliminary version of this work is presented in [25].

The paper is organized as follows. Preliminaries and definitions related to hybrid systems are given in Section 2. We explain the emulation approach for controller design in Section 3 and describe FlexRay in more detail in Section 4. We present the hybrid model in Section 5. The stability results are stated in Section 6 and an example is proposed in Section 7. Conclusions are given in Section 8. Most proofs are postponed to the Appendix.

\section{Preliminaries}

Let $\mathbb{Z}_{>0}:=\{1,2, \cdots\}, \mathbb{Z}_{\geq 0}:=\{0,1,2, \cdots\}$ and $\mathbb{R}_{\geq 0}:=$ $[0, \infty)$. Let $|x|$ denote the Euclidean norm of the vector $x \in \mathbb{R}^{n}$ and $I_{n}$ be the identity matrix of dimension $n$. For $(x, y) \in \mathbb{R}^{n+m},(x, y)$ stands for $\left[x^{T}, y^{T}\right]^{T}$. Given a closed set $\mathcal{A} \subset \mathbb{R}^{n}$ and $x \in \mathbb{R}^{n}$, we define the distance of a vector $x$ to $\mathcal{A}$ as $|x|_{\mathcal{A}}:=\inf _{y \in \mathcal{A}}|x-y|$. A setvalued mapping $M: \mathbb{R}^{m} \rightrightarrows \mathbb{R}^{n}$ is locally bounded at $x \in \mathbb{R}^{m}$ if there exists a neighborhood $U_{x}$ of $x$ such that $M\left(U_{x}\right) \subset \mathbb{R}^{n}$ is bounded. The mapping $M$ is locally bounded if it is locally bounded at each $x \in \mathbb{R}^{m}$. A setvalued mapping $M: \mathbb{R}^{m} \rightrightarrows \mathbb{R}^{n}$ is outer semi-continuous (OSC) if and only if its graph $\left\{(y, z): y \in \mathbb{R}^{m}, z \in\right.$ $M(y)\}$ is closed, see Lemma 5.10 in [4]. A continuous function $\gamma: \mathbb{R}_{\geq 0} \rightarrow \mathbb{R}_{\geq 0}$ is of class- $\mathcal{K}$ if it is zero at zero and strictly increasing and it is of class- $\mathcal{K}_{\infty}$ if, in addition, it is unbounded. A continuous function $\gamma$ : $\mathbb{R}_{\geq 0}^{2} \rightarrow \mathbb{R}_{\geq 0}$ is of class- $\mathcal{K} \mathcal{L}$ if for each $r \in \mathbb{R}_{\geq 0}, \gamma(\cdot, r)$ is of class- $\mathcal{K}$, and, for each $s \in \mathbb{R}_{\geq 0}, \gamma(s, \cdot)$ is decreasing to zero. For $x, v \in \mathbb{R}^{n}$ and $U: \mathbb{R}^{n} \rightarrow \mathbb{R}$, let $U^{\circ}(x ; v)$ be the Clarke's derivative of the function $U$ at $x$ in the direction $v: U^{\circ}(x ; v):=\lim \sup _{y \rightarrow x, \lambda \downarrow 0} \frac{U(y+\lambda v)-U(y)}{\lambda}$.

We will show that NCS with FlexRay can be represented by the following hybrid system ([4])

$$
\mathcal{H}:\left\{\begin{aligned}
\dot{\xi} & =\mathcal{F}(\xi) \xi \in C \\
\xi^{+} & =\mathcal{G}(\xi) \xi \in D
\end{aligned}\right.
$$

with state $\xi \in \mathbb{R}^{n}$ and where $C, D \subset \mathbb{R}^{n}$ are respectively the flow and the jump sets. We assume that the following basic assumptions ([4]) hold for system $\mathcal{H}$ :

(i) The sets $C$ and $D$ are subsets of $\mathbb{R}^{n}$ and are closed.

(ii) The mapping $\mathcal{F}$ is OSC and locally bounded, and $\mathcal{F}(\xi)$ is nonempty and convex for each $\xi \in C$.

(iii) The mapping $\mathcal{G}$ is OSC and locally bounded, and $\mathcal{G}(\xi)$ is nonempty for each $\xi \in D$.

These conditions ensure the well-posedness of system (1), see Chapter 6 in [4] for more details. These conditions are satisfied by the hybrid model we will construct. We now recall some definitions from [4]. A set $S \subset \mathbb{R}_{\geq 0} \times \mathbb{Z}_{\geq 0}$ is called a compact hybrid time domain if $S=\bigcup_{j=0}^{J-1}\left(\left[t_{j}, t_{j+1}\right], j\right)$ for some finite sequence of times $0=t_{0} \leq t_{1} \leq t_{2} \leq \cdots \leq t_{J}$. The set $S$ is a hybrid time domain if for all $(T, J) \in S, S \cap([0, T] \times\{0,1, \cdots, J\})$ is a compact hybrid time domain. A function $\xi: S \rightarrow \mathbb{R}^{n}$ is a hybrid arc if $S$ is a hybrid time domain and $\xi(\cdot, j)$ is locally absolutely continuous for each $j$. A hybrid arc $\xi: \operatorname{dom} \xi \rightarrow \mathbb{R}^{n}$ is a solution to the hybrid system $\mathcal{H}$ if $\xi(0,0) \in C \cup D$ and

(1) for all $j \in \mathbb{Z}_{\geq 0}$ and almost all $t$ such that $(t, j) \in$ $\operatorname{dom} \xi, \xi(t, j) \in C$ and $\dot{\xi}(t, j) \in \mathcal{F}(\xi(t, j))$; 
(2) for all $(t, j) \in \operatorname{dom} \xi$ such that $(t, j+1) \in \operatorname{dom} \xi$, $\xi(t, j) \in D$ and $\xi(t, j+1)=\mathcal{G}(\xi(t, j))$.

A solution is maximal if it cannot be extended. and it is complete when $\operatorname{dom} \xi$ is unbounded. We also recall the definition of uniform global pre-asymptotical stability (UGpAS) of a closed set for system (1), see Definition 3.6 and Theorem 3.40 in [4].

Definition 1 Consider system (1), the closed set $\mathcal{A} \subset$ $\mathbb{R}^{n}$ is $U G p A S$ if there exists $\beta \in \mathcal{K} \mathcal{L}$ such that all solutions $\xi$ satisfy

$$
|\xi(t, j)|_{\mathcal{A}} \leq \beta\left(|\xi(0,0)|_{\mathcal{A}}, t+j\right) \quad \forall(t, j) \in \operatorname{dom} \xi .
$$

Note that UGpAS implies that system $\mathcal{H}$ may generate maximal solutions, which are not complete.

\section{$3 \quad$ Emulation for NCS}

The purpose of this section is twofold. First, we explain the emulation method for controller design for NCS as proposed in $[1,14,15]$ that we follow in this paper. Second, we present an impulsive model and a hybrid model of NCS that was considered in prior work $[1,14,15]$ and explain why that the modeling approach is also pertinent to NCS with FlexRay. Our main observation is that NCS models considered in $[1,14,15]$ are not general enough to cover NCS with FlexRay and, hence, prior results cannot be used directly for this case. Therefore, in the rest of the paper, we first develop a high fidelity model of NCS with FlexRay and then develop new emulation results for this important case.

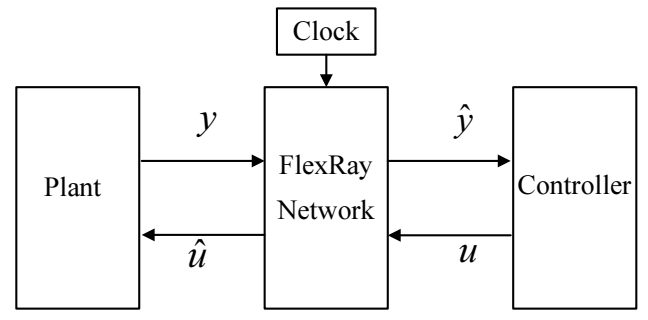

Fig. 1. Block diagram of NCS over FlexRay

The first step in the emulation approach is to design a controller for the plant while ignoring the network. The controller can be designed using any of the available nonlinear control techniques for continuous-time systems, such as those in e.g., $[9,10]$ and the references cited therein. Consider the plant model

$$
\dot{x}_{p}=f_{p}\left(x_{p}, u\right) \quad y=g_{p}\left(x_{p}\right),
$$

where $x_{p} \in \mathbb{R}^{n_{p}}$ is the state, $u \in \mathbb{R}^{n_{u}}$ is the control input and $y \in \mathbb{R}^{n_{y}}$ is the measured output. We suppose that the following controller has been designed for (3) while ignoring the network

$$
\dot{x}_{c}=f_{c}\left(x_{c}, y\right) \quad u=g_{c}\left(x_{c}\right),
$$

where $x_{c} \in \mathbb{R}^{n_{c}}$ is the state of the controller. The goal is to implement the designed controller over FlexRay network, as illustrated in Figure 1, and to demonstrate that under reasonable assumptions, the assumed stability of system (3)-(4) will be preserved for the NCS if data is transmitted sufficiently frequent over the network.

As shown in [14], the implementation of the controller (4) over a digital network leads to the following dynamics for almost all time between two successive transmissions, as in $[1,14,15]$,

$$
\begin{array}{ll}
\dot{x}_{p}=f_{p}\left(x_{p}, \hat{u}\right) & t \in\left[t_{i}, t_{i+1}\right] \\
\dot{x}_{c}=f_{c}\left(x_{c}, \hat{y}\right) & t \in\left[t_{i}, t_{i+1}\right] \\
\dot{\hat{y}}=\hat{f}_{p}\left(x_{p}, x_{c}, \hat{y}, \hat{u}\right) & t \in\left[t_{i}, t_{i+1}\right] \\
\dot{\hat{u}}=\hat{f}_{c}\left(x_{p}, x_{c}, \hat{y}, \hat{u}\right) & t \in\left[t_{i}, t_{i+1}\right] \\
y=g_{p}\left(x_{p}\right) & \\
u=g_{c}\left(x_{c}\right) &
\end{array}
$$

where the data transmission instants $t_{i}, i \in \mathbb{Z}_{>0}$, depend on the network. We have that $0<\varepsilon \leq t_{i+1}-t_{i} \leq \tau_{\text {MATI }}$, where $\tau_{\text {MATI }}>0$ is a measure of the network bandwidth and it would typically need to be small in the emulation method, and $\varepsilon$ represents the minimum achievable transmission interval given by the hardware constraints. The variables $\hat{u} \in \mathbb{R}^{n_{u}}$ and $\hat{y} \in \mathbb{R}^{n_{y}}$ respectively denote the networked versions of $u$ and $y$ available at the plant and the controller. Between the transmission instants, $\hat{y}$ and $\hat{u}$ are generated according to the in-network processing implementations defined by $\hat{f}_{p}$ and $\hat{f}_{c}$ in (5). Note that all the arguments of the functions $\hat{f}_{p}$ and $\hat{f}_{c}$ might not be available in all nodes. They are presented as in (5) for the sake of generality. Different implementations lead to different algorithms. For instance, the implementation of the controller using zero-order-hold devices gives $\hat{f}_{p}=0$ and $\hat{f}_{c}=0$.

We also introduce the network-induced error $e:=$ $\left(e_{y}, e_{u}\right)$ with $e_{y}:=\hat{y}-y$ and $e_{u}:=\hat{u}-u$. The error vector $e$ is partitioned as $e=\left(e_{1}, e_{2} \cdots e_{\ell}\right)$ according to the $\ell$ nodes (after reordering, if necessary). A node here refers to a group of sensor/actuator signals which are always transmitted in a single packet. Only one node is granted access to the communication channel at each transmission instant. The following assumption is useful for modeling network protocols ${ }^{1}$.

\footnotetext{
$\overline{1}$ This assumption can be relaxed as demonstrated in [13].
} 
Assumption 1 If the $s$-th node, for $s \in\{1, \cdots, \ell\}$, gets access to the network at some transmission time $t_{i}$, we have $e_{s}\left(t_{i}^{+}\right)=0$.

Using Assumption 1, it was shown in [14] that a large class of network protocols can be modeled as

$$
e\left(t_{i}^{+}\right)=h\left(i, e\left(t_{i}\right)\right)
$$

where $h(\cdot, \cdot)$ depends only on the network protocol and is independent of the plant and controller dynamics. We present the map $h(\cdot, \cdot)$ for two such protocols that were modeled for the first time in form (6) in [14]: Round Robin (RR $)^{2}$ and Try Once Discard (TOD) ${ }^{3}$ protocols. These protocols are important for this paper since we will show that FlexRay allows us to switch between these two protocols; this situation is analysed in detail in the sequel. Using Assumption 1, we can write the model of $\mathrm{RR}$ protocol as follows

$$
h_{\mathrm{RR}}(i, e):=(I-\Delta(i)) e,
$$

with $\Delta(i)=\operatorname{diag}\left\{\delta_{1}(i) I_{n_{1}}, \cdots, \delta_{\ell}(i) I_{n_{\ell}}\right\}, \delta_{s}(i)=1$ when $s-1=i \bmod \ell$ and $\delta_{s}(i)=0$ otherwise, $n_{s} \in \mathbb{Z}_{>0}$ and satisfies $\sum_{s=1}^{\ell} n_{s}=n_{e}$. The model of TOD protocol takes the form

$$
h_{\mathrm{TOD}}(e):=(I-\psi(e)) e,
$$

where $\psi(e):=\operatorname{diag}\left\{\psi_{1}(e) I_{n_{1}}, \psi_{2}(e) I_{n_{2}}, \cdots, \psi_{\ell}(e) I_{n_{\ell}}\right\}$, $\psi_{s}(e)=1$ when $s=\min \left(\arg \max _{j \in\{1, \cdots, \ell\}}\left|e_{j}\right|\right)$ and $\psi_{s}(e)=0$ otherwise, for $s \in\{1, \ldots, \ell\}$ and $i \in \mathbb{Z}_{\geq 0}$.

An impulsive model for NCS with scheduling protocols of the form (6) is given by ([14])

$$
\begin{array}{ll}
\dot{x}=f(x, e) & t \in\left[t_{i}, t_{i+1}\right] \\
\dot{e}=g(x, e) & t \in\left[t_{i}, t_{i+1}\right] \\
x\left(t_{i}^{+}\right)=x\left(t_{i}\right) & \\
e\left(t_{i}^{+}\right)=h\left(i, e\left(t_{i}\right)\right) &
\end{array}
$$

where $x:=\left(x_{p}, x_{c}\right)$. The expressions of $f$ and $g$ are obtained by direct calculations from (3) and (4), see [14]. Furthermore, it was shown in [1] that the above impulsive model can be embedded in a hybrid model of the

\footnotetext{
2 RR protocol assigns access to network in a predetermined and cyclic manner.

3 TOD protocol gives access to the node with the largest mismatch between the current signal value and the last transmitted one, see [23].
}

form of (1) that is more amenable for analysis

$$
\left.\begin{array}{l}
\dot{x}=f(x, e) \\
\dot{e}=g(x, e) \\
\dot{\tau}=1 \\
\dot{\kappa}=1
\end{array}\right\} \tau \in\left[0, \tau_{\mathrm{MATI}}\right]
$$

The clock variable $\tau \in \mathbb{R}_{>0}$ is used to generate jumps in the hybrid model that correspond to data transmissions and the variable $\kappa \in \mathbb{Z}_{\geq 0}$ counts the number of transmissions that is needed to implement certain protocols, such as Round Robin.

It turns out that the models (9) and (10) are not general enough to cover the case of NCS with FlexRay because of the switches incurred by the scheduling rules. We develop more general impulsive and hybrid models for NCS with FlexRay in the following sections.

\section{FlexRay Network}

In this section, we provide a detailed description of the FlexRay communication cycle and introduce the modeling assumptions that allow us to represent NCS with FlexRay as a hybrid system of the form (1).

\subsection{Communication cycle}

We investigate the scenario where a FlexRay network is used to connect the plant and the controller, as depicted in Figure 1. The vector $(u, y)$ in Figure 1 is partitioned into nodes. Data is coded into packets and a packet is sent at each transmission instant $t_{i}^{s}$ and is received at a reception instant $t_{i}^{r}$ for $i \in \mathbb{Z}_{>0}$, as illustrated in Figure 2 . The transmission time $t_{i}^{r}-t_{i}^{s}$ depends on the size of the packet: the larger the packet, the longer the transmission time.

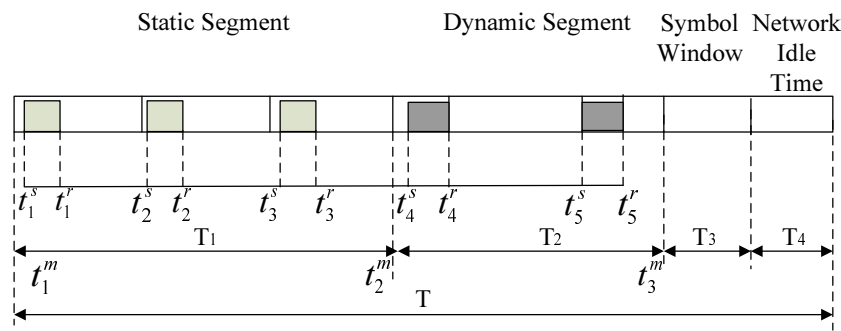

Fig. 2. FlexRay communication cycle 
FlexRay is characterized by pre-set communication cycles of length $T>0$, see [3]. Each cycle contains a static segment, a dynamic segment and two protocol segments called a symbol window and a network idle time, which are of respective lengths $T_{1}, T_{2}, T_{3}$ and $T_{4}>0$, see Figure 2. Distinct network access techniques are applied for the static and the dynamic segments $[3,8,16]$. In particular, the static segment relies on a time division multiple access (TDMA) approach and the network capacity is assigned to the nodes by a static protocol such as RR. The dynamic segment employs the flexible time division multiple access (FTDMA) technique [2], which enables nodes to compete for accessing the network and a dynamic protocol such as TOD can be implemented to select nodes to transmit messages based on the online information. The symbol window is a time slot for transmitting data for network management purposes and the network idle time is a communication-free period that is required for clock synchronization. Compared to the lengths of the static and the dynamic segments, the lengths of the symbol window and the network idle time are small [3]. Consequently, we make the following assumption.

Assumption 2 The lengths of the symbol window and the network idle time are negligible compared to $T_{1}$ and $T_{2}$, hence $T_{3}=T_{4}=0$.

A consequence of Assumption 2 is that $T=T_{1}+T_{2}$. Note that the length of the cycle as well as $T_{1}$ and $T_{2}$ can be configured but are fixed once the network is implemented [3]. Let $t_{j}^{m}, j \in \mathbb{Z}_{>0}$ denote the segments switching instants; note that prior work in $[1,14]$ did not require this level of complexity to model the network. Assume that FlexRay cycles start with the static segment without loss of generality, as in Figure 2 (this assumption will be later relaxed). It is very useful to introduce a state variable $q \in\{1,2\}$ which keeps track of whether we are in the static segment $(q=1)$ or in the dynamic segment $(q=2)$. Hence, we can write

$$
\begin{array}{ll}
q(t)=1, \quad \forall t \in\left[t_{j}^{m}, t_{j}^{m}+T_{1}\right), \quad j=2 n-1 \\
q(t)=2, \quad \forall t \in\left[t_{j}^{m}, t_{j}^{m}+T_{2}\right), \quad j=2 n
\end{array}
$$

for all $n \in \mathbb{Z}_{>0}$. In order to impose this constraint on our model in a manner consistent with the hybrid model (1), we claim that (11) can be equivalently be written as

$$
\begin{aligned}
& \left.\dot{\tau}_{2}=1\right\} \\
& \dot{q}=0\} \\
& \left(\tau_{2}, q\right) \in\left(\left[0, T_{1}\right] \times\{1\}\right) \cup\left(\left[0, T_{2}\right] \times\{2\}\right) \\
& \left.\begin{array}{l}
\tau_{2}^{+}=0 \\
q^{+}=3-q
\end{array}\right\} \\
& \left(\tau_{2}, q\right) \in\left(\left\{T_{1}\right\} \times\{1\}\right) \cup\left(\left\{T_{2}\right\} \times\{2\}\right),
\end{aligned}
$$

where $\tau_{2} \in \mathbb{R}_{\geq 0}$ is a clock which corresponds to the time elapsed since the last segment switch. If we interpret the sequence of switching times for this hybrid model as $t_{i}^{m}$, we can see that during the time intervals $\left[t_{i}^{m}, t_{i+1}^{m}\right]$ we have that $q(t, i)$ is a constant in $\{1,2\}$ and $\tau_{2}(t, i)=$ $t-t_{i}^{m}$. Switches can only occur when $\left(\tau_{2}, q\right)=\left(T_{1}, 1\right)$ or $\left(\tau_{2}, q\right)=\left(T_{2}, 2\right)$. At every switch, $q$ toggles between 1 and 2 whereas $\tau_{2}$ is reset to zero. This implies that (11) holds if $q(0,0)=1$. Note that the hybrid model also covers the case when $q(0,0)=2$, which relaxes our assumption that cycles starts with the static segment.

We next focus on modeling the data transmissions during the static and the dynamic segments.

\subsection{Static segment}

The static segment consists of time slots of equal length $\tau_{s t}>0$, as illustrated in Figure 3 . At the beginning of each time slot, a single node is chosen by the protocol, codes its data and sends it over the communication channel. The coding time is denoted by $\mu_{i}^{s t}, i \in \mathbb{Z}_{>0}$.



Fig. 3. Static segment

We use the following assumption.

\section{Assumption 3}

(a) Data are transmitted instantaneously, i.e. $t_{i}:=t_{i}^{s}=$ $t_{i}^{r}$ for all $i \in \mathbb{Z}_{>0}$; data coding time is negligible, i.e. $\mu_{i}^{s t}=0$ for all $i \in \mathbb{Z}_{>0}$.

(b) A transmission occurs at the end of all the static segments.

(c) All static time slots are of equal length $\tau_{\text {st }}>0$.

Item (a) is reasonable when the packets are short. Otherwise, the effects of transmission time $t_{i}^{r}-t_{i}^{s}$ and coding time $\mu_{i}^{s t}$ can be modeled as a time-delay and we believe that the results presented hereafter can be extended to cover this case using [6]. We do not consider this generalization here to concentrate on the challenges due to the switches between the static and dynamic segments. Item (b) can be enforced in practice. Item (c) comes from the FlexRay configuration, see Chapter 9.1 in [3].

According to Assumption 3, the MATI during the static segment is given by the length of the time slots $\tau_{s t}$, hence we let $\tau_{\text {MATI }}^{s}:=\tau_{s t}$. Note that item (c) ensures that for any given $T_{1}>0$, there exists $k \in \mathbb{Z}_{>0}$ such that 
$T_{1}=k \tau_{\text {MATI }}^{s}$ holds. The lemma below follows directly from Assumption 3.

Lemma 1 Suppose Assumption 3 holds. Then the transmission instants sequence $\left\{t_{i}\right\}_{i \in \mathbb{Z}_{>0}}$, satisfies

$$
t_{i+1}-t_{i}=\tau_{\text {MATI }}^{s} \quad \forall t_{i}, t_{i+1} \in\left[t_{2 n-1}^{m}, t_{2 n}^{m}\right),
$$

where $n \in \mathbb{Z}_{>0}$ and the segment switching instants sequence $t_{j}^{m}, j \in \mathbb{Z}_{>0}$, satisfies (11).

In order to develop a hybrid model of NCS with FlexRay in the formalism of [4], we introduce a clock variable $\tau_{1}$ which describes the time elapsed since the last transmission, as $\tau$ does in (10). This variable has the following dynamics during the static segment.

$$
\begin{array}{ll}
\dot{\tau}_{1}=1 & \left(\tau_{1}, \tau_{2}, q\right) \in C_{1} \\
\tau_{1}^{+}=0 & \left(\tau_{1}, \tau_{2}, q\right) \in D_{1}^{\text {trans }}
\end{array}
$$

with

$$
\begin{aligned}
C_{1} & :=\left[0, \tau_{\text {MATI }}^{s}\right] \times\left[0, T_{1}\right] \times\{1\} \\
D_{1}^{\text {trans }} & :=\left\{\tau_{\text {MATI }}^{s}\right\} \times\left[0, T_{1}\right] \times\{1\} .
\end{aligned}
$$

Equations (15) and (16) simply mean that the transmission clock $\tau_{1}$ is reset to 0 when it reaches $\tau_{\text {MATI }}^{s}$ during the static segment, i.e. when $q=1$ and $\tau_{2} \in\left[0, T_{1}\right]$. It is not hard to check that the switching time instants $t_{i}$, $i \in \mathbb{Z}_{>0}$, for the solutions of the hybrid system (15) satisfy the constraint in (14) and are indeed consistent with Assumption 3.

As mentioned before, a static TDMA scheme is used to arbitrate the transmission during the static segment. The length of static time slots is first configured and the latter are assigned to the nodes in a predetermined way. As a consequence, we can only implement static protocols, such as the RR protocol, during the static segments. In this case, the protocol equation during the static segment is given by (7).

\subsection{Dynamic segment}

The dynamic segments are composed of minislots, which are substantially shorter than the static time slots and do not necessarily correspond to the transmission of a packet. Data is coded into packets before being sent to the physical channel and to transmit a packet typically requires more than one minislot. Nodes compete to transmit their packets and the scheduling rule associated to the dynamic segment grants the priority based on the online information. The node with the highest priority is assigned with the 'earliest' minislot, that is the minislot with the lowest number. An illustration is given in Figure 4, where the minislots indexed by 2,3 and 11 to
13 are dedicated to transmissions. On the other hand, the minislots are idle when no nodes compete for access; see the minislots indexed by 1, 4 to 10 and 14 to 23 in Figure 4 . We denote by $\tau_{d y, i}, i \in \mathbb{Z}_{>0}$, the times during which the network is idle and we introduce $\mu_{j}^{d y}, j \in \mathbb{Z}_{>0}$ to represent the data coding time.

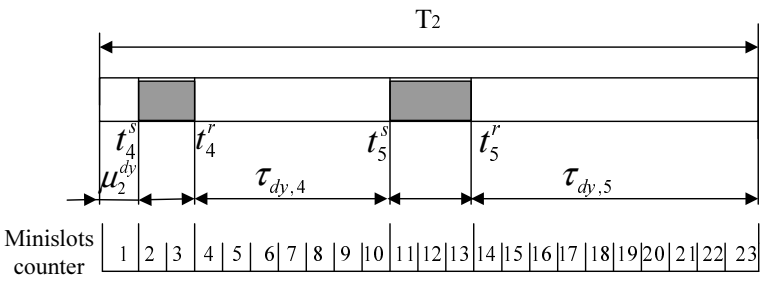

Fig. 4. Dynamic segment and minislots

For modeling purposes, we summarize the assumptions we make on the dynamic segment.

\section{Assumption 4}

(a) Data are transmitted instantaneously, i.e. $t_{i}:=t_{i}^{s}=$ $t_{i}^{r}$ for all $i \in \mathbb{Z}_{>0}$; data coding time is negligible, i.e. $\mu_{i}^{d y}=0$ for all $i \in \mathbb{Z}_{>0}$.

(b) A transmission occurs at the end of all the dynamic segments.

(c) The dynamic segments satisfy $\tau_{d y, i} \in\left[\varepsilon, \tau_{\text {MATI }}^{d}\right]$ for some $\tau_{\text {MATI }}^{d}>0$ and for all $i \in \mathbb{Z}_{>0}$, where $\varepsilon>0$ refers to the length of a minislot.

Items (a) corresponds to item (a) of Assumption 3. Item (b) can be enforced by design, see Remark 1 for more detail. Item (c) of Assumption 4 is justified by the fact that, when a node has been granted access, the other ones need to wait for at least the next minislot of length $\varepsilon$ to possibly transmit. Furthermore, we suppose that the network is such that two transmissions occur at most every $\tau_{\text {MATI }}^{d}$ units of time. The lemma below follows from Assumption 4.

Lemma 2 Suppose Assumption 4 holds. Then the transmission instants sequence $\left\{t_{i}\right\}_{i \in \mathbb{Z}>0}$, satisfies

$$
t_{i+1}-t_{i} \in\left[\varepsilon, \tau_{\text {MATI }}^{d}\right] \quad \forall t_{i}, t_{i+1} \in\left[t_{2 n}^{m}, t_{2 n+1}^{m}\right)
$$

where $n \in \mathbb{Z}_{>0}$ and the segment switching instants sequence $t_{j}^{m}, j \in \mathbb{Z}_{>0}$, satisfies (11).

Proof. Assumption 2 with (11) shows that $\left[t_{2 n-1}^{m}, t_{2 n}^{m}\right]$ and $\left[t_{2 n}^{m}, t_{2 n+1}^{m}\right]$ for $n \in \mathbb{Z}_{>0}$ respectively describe the static and the dynamic segments. Item (a) of Assumption 4 implies that $\tau_{d y, i}$ denotes the (maximum) intertransmission time interval for these segments. Consequently, we deduce that the transmission instants satisfy (17) using items (b) and (c) of Assumption 4.

Remark 1 Two successive transmissions are spaced by $\varepsilon$ units of time, in view of item (c) of Assumption 4 . 
The assumption that a transmission occurs at the end of the segments therefore excludes the case when a packet is transmitted during the last minislot of the dynamic segment, which is in agreement with how FlexRay works [19]. This approximation is fine as $\varepsilon$ is typically very small. Hence, if a transmission does not occur at the very end of the dynamic segment but $\varepsilon$ units of time before, that will induce a small delay, which we neglect.

The variable $\tau_{1}$ introduced in Section 4.2 has the following dynamics during the dynamic segment in view of Assumption 4.

$$
\begin{array}{ll}
\dot{\tau}_{1}=1 & \left(\tau_{1}, \tau_{2}, q\right) \in C_{2} \\
\tau_{1}^{+}=0 & \left(\tau_{1}, \tau_{2}, q\right) \in D_{2}^{\text {trans }}
\end{array}
$$

with

$$
\begin{aligned}
C_{2} & :=\left[0, \tau_{\text {MATI }}^{d}\right] \times\left[0, T_{2}\right] \times\{2\} \\
D_{2}^{\text {trans }} & :=\left[\varepsilon, \tau_{\text {MATI }}^{d}\right] \times\left[0, T_{2}\right] \times\{2\}
\end{aligned}
$$

Contrary to the static segment, transmissions do not a priori occur periodically in the dynamic segment, which justifies the fact that both flow and jump are allowed when $\tau_{1} \in\left[\varepsilon, \tau_{\text {MATI }}^{d}\right]$ as in (10). It has to be noted that if a solution to $(18),(19)$ becomes such that $\tau_{1} \in[0, \varepsilon)$ and $\tau_{2}=T_{2}$, it stops to exist. This is not an issue because, first, the stability property we guarantee in Section 6 still holds in this case; second, because this situation does not occur in practice according to Remark 1.

The FTDMA scheme is employed to arbitrate the transmission during the dynamic segment, which enables packets to be sent whenever required, so that only dynamic protocols like TOD can be implemented. In this case, the protocol equation during the dynamic segment is given by (8).

\subsection{Protocol equation}

In view of Sections 4.2 and 4.3 , we propose the following equation to model the protocol dynamics

$$
e\left(t_{i}^{+}\right)=h\left(i, e\left(t_{i}\right), q\left(t_{i}\right)\right)
$$

We see that we need to make the mapping $h$ depend on the variable $q$, which indicates the active segment. This is again justified by the fact that different protocols are used during the static and the dynamic segments. The mapping $h$ can be further written as

$$
h(i, e, q):=(2-q) h_{s}(i, e)+(q-1) h_{d}(i, e),
$$

where $h_{s}$ and $h_{d}$ are defined by the scheduling rules of the static and the dynamic segments, respectively. In that way, the variable $q$ generates switches of the scheduling policy between $h_{s}$ and $h_{d}$; that is, $h(i, e, 1)=h_{s}(i, e)$ and $h(i, e, 2)=h_{d}(i, e)$. Examples include $h_{s}=h_{\mathrm{RR}}$ and $h_{d}=h_{\mathrm{TOD}}$ where $h_{\mathrm{RR}}$ and $h_{\mathrm{TOD}}$ are respectively defined in (7) and (8).

\section{$5 \quad$ Hybrid model of NCS with FlexRay}

\subsection{Impulsive model}

In view of Section 4 , NCS with FlexRay can be modeled by the impulsive system below under Assumptions 2-4,

$$
\begin{array}{ll}
\dot{x}=f(x, e) & t \in\left[t_{i}, t_{i+1}\right] \\
\dot{e}=g(x, e) & t \in\left[t_{i}, t_{i+1}\right] \\
\dot{q}=0 & t \in\left[t_{j}^{m}, t_{j+1}^{m}\right] \\
x\left(t_{i}^{+}\right)=x\left(t_{i}\right) & \\
e\left(t_{i}^{+}\right)=h\left(i, e\left(t_{i}\right), q\left(t_{i}\right)\right) & \\
q\left(t_{i}^{+}\right)=q\left(t_{i}\right) & \\
x\left(t_{j}^{m+}\right)=x\left(t_{j}^{m}\right) & \\
e\left(t_{j}^{m+}\right)=e\left(t_{j}^{m}\right) & \\
q\left(t_{j}^{m+}\right)=3-q\left(t_{j}^{m}\right) &
\end{array}
$$

where $x:=\left(x_{p}, x_{c}\right) \in \mathbb{R}^{n_{x}}, i, j, m \in \mathbb{Z}_{>0}$, the vector fields $f$ and $g$ are obtained by direct calculations from (3) and (4) and are assumed to be continuous. We also assume that $h$ is continuous, which is the case for RR and TOD protocols in view of (7) and (8), respectively. Compared to the impulsive model for non-switched protocol in (9), system (22) is subject to two types of jumps, namely, the transmission jumps in (22b) and the segment switching jumps in $(22 \mathrm{c})$. In addition, we need to introduce the variable $q$ as explained in Section 4.1, and the protocol equation is also different.

\subsection{Hybrid model in the formalism of [4]}

In order to proceed with the analysis, we map the model (22) into a hybrid system using the formalism of [4]. We introduce the counter variable $\kappa \in \mathbb{Z}_{\geq 0}$ as in (10) and let $\xi:=\left(x, e, \kappa, \tau_{1}, \tau_{2}, q\right)$. Using (15), (18) and (21), we model NCS with FlexRay as

$$
\begin{aligned}
\dot{\xi} & =\mathcal{F}(\xi) & & \xi \in C \\
\xi^{+} & =\mathcal{G}(\xi) & & \xi \in D
\end{aligned}
$$

where

$$
\begin{aligned}
& C:=\mathbb{R}^{n_{x}} \times \mathbb{R}^{n_{e}} \times \mathbb{Z}_{\geq 0} \times\left(C_{1} \cup C_{2}\right) \\
& D:=\mathbb{R}^{n_{x}} \times \mathbb{R}^{n_{e}} \times \mathbb{Z}_{\geq 0} \times\left(D_{1} \cup D_{2}\right)
\end{aligned}
$$


with $C_{1}$ and $C_{2}$ coming respectively from (15) and (18), $D_{1}:=D_{1}^{\text {trans }} \cup D_{1}^{\text {seg }}, D_{2}:=D_{2}^{\text {trans }} \cup D_{2}^{\text {seg }}$ with $D_{1}^{\text {trans }}$ and $D_{2}^{\text {trans }}$ defined in (15) and (18) respectively, and

$$
\begin{aligned}
& D_{1}^{\text {seg }}:=\{0\} \times\left\{T_{1}\right\} \times\{1\} \\
& D_{2}^{\text {seg }}:=\{0\} \times\left\{T_{2}\right\} \times\{2\}
\end{aligned}
$$

The mapping $\mathcal{F}$ in (23) is defined as

$$
\mathcal{F}(\xi):=(f(x, e), g(x, e), 0,1,1,0)
$$

and the mapping $\mathcal{G}$ is given by

$$
\mathcal{G}(\xi):= \begin{cases}\mathcal{G}^{\text {trans }}(\xi) & \left(\tau_{1}, \tau_{2}, q\right) \in D_{1}^{\text {trans }} \cup D_{2}^{\text {trans }} \\ \mathcal{G}^{\text {seg }}(\xi) & \left(\tau_{1}, \tau_{2}, q\right) \in D_{1}^{\text {seg }} \cup D_{2}^{\text {seg }}\end{cases}
$$

where $\mathcal{G}^{\text {trans }}(\xi):=\left(x, h(\kappa, e, q), \kappa+1,0, \tau_{2}, q\right)$ corresponds to a transmission jump, $\mathcal{G}^{\mathrm{seg}}(\xi):=\left(x, e, \kappa, \tau_{1}, 0,3-\right.$ $q)$ corresponds to a segment switching jump. We can see that $\mathcal{G}$ generates two kinds of jumps whether it corresponds to a transmissions or a segment switch. Recall that a transmission jump is enforced at the end of segments from Assumptions 3-4, $\mathcal{G}^{\text {trans }}$ maps the vector $\left(\tau_{1}, \tau_{2}, q\right)$ to $\left(0, T_{1}, 1\right)$ or $\left(0, T_{2}, 2\right)$ when $\tau_{2}=T_{1}$ or $T_{2}$. Consequently, another jump will immediately occur as $\left(\tau_{1}, \tau_{2}, q\right)$ enters either $D_{1}^{\mathrm{seg}}$ or $D_{2}^{\mathrm{seg}}$. This captures the fact that two kinds of jumps occur simultaneously at the end of the segments. The hybrid system defined above includes all possible solutions that system (22) generates and it satisfies basic conditions listed in Section 2, as stated below.

Proposition 1 System (23) satisfies the basic assumptions defined in Section 2.

Proof. First, the sets $C$ and $D$ in (24) are closed. Second, the flow map $\mathcal{F}$ is continuous as so are $f$ and $g$ by assumption. Third, by defining the jump map $\mathcal{G}$ as $\emptyset$ on $\left(\mathbb{R}^{n_{x}+n_{e}} \times \mathbb{Z}_{\geq 0} \times \mathbb{R}_{>0}^{2} \times\{1,2\}\right) \backslash D$, we derive that $\mathcal{G}$ is OSC as its graph is closed. It is also locally bounded by continuity of $\mathcal{G}^{\text {trans }}$ and $\mathcal{G}^{\text {seg }}$ (recall that $h$ is assumed to be continuous) and $\mathcal{G}(\xi)$ is nonempty for each $\xi \in D$ in view of (27).

\section{$6 \quad$ Asymptotic stability properties}

In this section, we first state the assumptions we make on system (23). We then construct a novel Lyapunov function for the hybrid model (23) which is shown to decrease on flows and not to increase at jumps, from which we derive that a global asymptotic stability property holds for system (23).

\subsection{Assumptions}

We assume that, on each segment, the scheduling protocol is such that the following assumption holds.

Assumption 5 For each $q \in\{1,2\}$, there exist $W_{q}$ : $\mathbb{R}^{n_{e}} \times \mathbb{Z}_{\geq 0} \rightarrow \mathbb{R}_{\geq 0}$, which is locally Lipschitz in its first argument, a continuous function $H_{q}: \mathbb{R}^{n_{x}} \rightarrow \mathbb{R}_{\geq 0}$, constants $\underline{\alpha}_{W_{q}}, \bar{\alpha}_{W_{q}}>0, L_{q} \geq 0$ and $\rho_{q} \in[0,1)$ such that for any $\kappa \in \mathbb{Z}_{\geq 0}$ and $e \in \mathbb{R}^{n_{e}}$

(1) $\underline{\alpha}_{W_{q}}|e| \leq W_{q}(\kappa, e) \leq \bar{\alpha}_{W_{q}}|e|$,

(2) $W_{q}(\kappa+1, h(\kappa, e, q)) \leq \rho_{q} W_{q}(\kappa, e)$,

and for all $x \in \mathbb{R}^{n_{x}}, \kappa \in \mathbb{Z}_{\geq 0}$ and almost all $e \in \mathbb{R}^{n_{e}}$

(3) $\left\langle\frac{\partial W_{q}(\kappa, e)}{\partial e}, g(x, e)\right\rangle \leq L_{q} W_{q}(\kappa, e)+H_{q}(x)$.

Items (1)-(2) of Assumption 5 mean that the scheduling rules of each segment are uniformly globally exponentially stable (see [14]). As already pointed out, a possible choice is the RR protocol for the static segment and the TOD protocol for the dynamic segment, as given in (7) and (8). Item (3) of Assumption 5 is an exponential growth condition on the $e$-system. It is satisfied when $W$ is globally Lipschitz in $e$, which is the case of the $\mathrm{RR}$ and the TOD protocol in view of [14], and when $g(x, e) \leq M(x)+M|e|$ for any $(x, e) \in \mathbb{R}^{n_{x}+n_{e}}$, with $M>0$. In this case, item (3) of Assumption 5 holds with $L_{q}:=\frac{S_{q} M}{\underline{\alpha}_{W_{q}}}, H_{q}(x):=S_{q} M|x|$, where $S_{q}$ is the Lipschitz constant of $W_{q}$.

We also assume that controller (4) is designed such that the $x$-system in $(23)$ is $\mathcal{L}_{2}$ stable from $W_{q}$ to $H_{q}$ for $q \in\{1,2\}$. This robustness property is similar to the corresponding assumption in $[1,14]$, where non-switched protocols are considered. This assumption may be ensured at the first step of the emulation design by investigating the robustness of the closed-loop system (3), (4) to the measurement and the input disturbances.

Assumption 6 There exist a continuously differentiable function $V: \mathbb{R}^{n_{x}} \rightarrow \mathbb{R}_{\geq 0}$ and $\underline{\alpha}_{V}, \bar{\alpha}_{V} \in \mathcal{K}_{\infty}$ such that for each $q \in\{1,2\}$ there exist a positive definite continuous function $\varrho_{q}$ and $\gamma_{q}>0$ such that for all $x \in \mathbb{R}^{n_{x}}, e \in \mathbb{R}^{n_{e}}$ and $\kappa \in \mathbb{Z}_{\geq 0}$

$$
\begin{aligned}
\underline{\alpha}_{V}(|x|) & \leq V(x) \leq \bar{\alpha}_{V}(|x|) \\
\langle\nabla V(x), f(x, e)\rangle \leq & -\varrho_{q}(|x|)-\varrho_{q}(|e|) \\
& -H_{q}^{2}(x)+\gamma_{q}^{2} W_{q}^{2}(\kappa, e)
\end{aligned}
$$

where the functions $H_{q}$ and $W_{q}$ come from Assumption 5.

The last assumption requires sufficiently small MATIs during the static and dynamic segments. A feature of 
the problem is that the MATI bounds we propose below depend on the length of the segments.

We first introduce some notations for the ease of presentation. Let $\rho_{m} \in(0,1)$ and $\gamma_{m}>0$ come from Assumptions $5-6, \pi_{m}:=\bar{\alpha}_{W_{3-m}} / \underline{\alpha}_{W_{m}}$ for $m \in\{1,2\}$ and the function ${ }^{4} c_{m}: \mathbb{R}_{>0} \rightarrow \mathbb{R}_{>0}$ be defined by, for $s>0$,

$$
c_{m}(s):=\frac{\gamma_{m}}{\gamma_{m} / k_{m}-d_{m}(s)}+\delta_{m}+\frac{1}{\rho_{m} s},
$$

where

$$
\begin{aligned}
& \delta_{m}:=\frac{(M-1) \gamma_{m}}{\rho_{m}^{2}} \\
& d_{m}(s):= \begin{cases}\frac{k_{m} M-\gamma_{m}}{k_{m} \exp \left(k_{m} \rho_{m} s\right)} & \text { when } M \leq \frac{2 \gamma_{m}}{k_{m}} \\
\frac{\gamma_{m}}{k_{m} \exp \left(\frac{\gamma_{m} k_{m} \rho_{m}}{k_{m} M-\gamma_{m}} s\right)} & \text { when } M \geq \frac{2 \gamma_{m}}{k_{m}}\end{cases}
\end{aligned}
$$

with $M:=\max \left\{1, \frac{\gamma_{2} \rho_{1} \rho_{2}}{\gamma_{1} \pi_{2}^{2}}, \frac{\gamma_{1} \rho_{1} \rho_{2}}{\gamma_{2} \pi_{1}^{2}}\right\}, k_{m}:=\max \left\{\gamma_{m}\right.$, $\left.\frac{\gamma_{m}^{2} \pi_{3-m}^{2}}{\gamma_{3-m} \rho_{1} \rho_{2}}\right\}$.

Assumption 7 For each $T_{1}, T_{2}>0$,

$$
\tau_{\mathrm{MATI}}^{s}=\frac{T_{1}}{\left\lceil T_{1} / \tau_{\mathrm{MATI}, 1}\right\rceil} \quad \tau_{\mathrm{MATI}}^{d}=\tau_{\mathrm{MATI}, 2},
$$

where, for $m \in\{1,2\}$

$$
\begin{aligned}
& \tau_{\text {MATI }, m} \\
& = \begin{cases}\frac{1}{L_{m} r_{m}\left(T_{m}\right)} \arctan \left(\lambda\left(T_{m}\right)\right) & \sqrt{\gamma_{m} c_{m}\left(T_{m}\right)}>L_{m} \\
\frac{1}{\rho_{m} \gamma_{m}+L_{m}}-\frac{\rho_{m}}{\rho_{m} L_{m}+\gamma_{m}} & \sqrt{\gamma_{m} c_{m}\left(T_{m}\right)}=L_{m} \\
\frac{1}{L_{m} r_{m}\left(T_{m}\right)} \operatorname{arctanh}\left(\lambda\left(T_{m}\right)\right) & \sqrt{\gamma_{m} c_{m}\left(T_{m}\right)}<L_{m},\end{cases}
\end{aligned}
$$

with $L_{m}, \gamma_{m}>0$ and $\rho_{m} \in(0,1)$ coming from Assumptions 5 and $6, \lambda(s):=\frac{r_{m}(s)\left(1-\rho_{m}\right)}{2 \frac{\rho_{m}}{1+\rho_{m}}\left(\frac{\gamma_{m}}{L_{m}}-1\right)+1+\rho_{m}}, r_{m}(s):=$ $\sqrt{\left|\frac{\gamma_{m} c_{m}(s)}{L_{m}^{2}}-1\right|}$ and $c_{m}(s)$ come from (29) for $s>0$.

The MATIs in (31) depend on the constants introduced in Assumptions 5-6 as well as on the segment lengths. The first equation in (31) is justified by the fact that $T_{1}$ has to be a multiple of $\tau_{\text {MATI,1 }}$ according to Section 4.2. Compared to the MATI bounds in [1], the expression in (32) involve $c_{m}\left(T_{m}\right)$. The smaller $T_{m}$ the larger $c_{m}$, which leads to a smaller $\tau_{\text {MATI,m }}$. In particular, when $T_{1}$ or $T_{2}$ goes to infinity which corresponds to the case there exists only one segment, the MATI bounds in (31) tend to finite values which agree with the MATI bounds in [1], as explained below in Remark 2 .

\footnotetext{
4 Note that $c_{m}$ in (29) is a strictly positive function since it decreases when its argument grows and $c_{m}(s) \rightarrow k_{m}+\delta_{m}>0$ when $s \rightarrow \infty$.
}

\subsection{Hybrid Lyapunov function and stability results}

Based on the assumptions listed above, we can study the stability of system (23) using the following Lyapunov function

$$
\begin{aligned}
U(\xi):= & V(x)+\gamma_{1} \phi_{1}\left(\tau_{1}\right) \theta_{1}\left(\tau_{2}\right) W_{1}^{2}(\kappa, e)(2-q) \\
& +\gamma_{2} \phi_{2}\left(\tau_{1}\right) \theta_{2}\left(\tau_{2}\right) W_{2}^{2}(\kappa, e)(q-1),
\end{aligned}
$$

for any $\xi \in C \cup D$, where $V, W_{1}$ and $W_{2}$ come from assumptions stated in the previous section. The scalar functions $\phi_{1}, \phi_{2}, \theta_{1}$ and $\theta_{2}$ are designed in the Appendix in such a way that the following proposition holds.

Proposition 2 Consider system (23), suppose Assumptions 5-7 hold and let $\mathcal{A}:=\{\xi: x=0, e=0\}$. Then, there exist $\underline{\alpha}_{U}, \bar{\alpha}_{U} \in \mathcal{K}_{\infty}$ and a continuous positive definite function $\varrho: \mathbb{R}_{\geq 0} \rightarrow \mathbb{R}_{\geq 0}$ such that the following holds for the function $U$ in (33).

(1) $U$ is locally Lipschitz in $x, e, \tau_{1}$ and $\tau_{2}$.

(2) For all $\xi \in C \cup D, \underline{\alpha}_{U}\left(|\xi|_{\mathcal{A}}\right) \leq U(\xi) \leq \bar{\alpha}_{U}\left(|\xi|_{\mathcal{A}}\right)$.

(3) For all $\xi \in C, U^{\circ}(\xi ; \mathcal{F}(\xi)) \leq-\varrho\left(|\xi|_{\mathcal{A}}\right)$.

(4) For all $\xi \in D, U(\mathcal{G}(\xi)) \leq U(\xi)$.

Proposition 2 allows to derive the following stability result for the set $\mathcal{A}$.

Theorem 1 Consider system (23) and suppose Assumptions 5-7 hold, then the set $\mathcal{A}=\{\xi: x=0, e=0\}$ is $U G p A S$.

Remark 2 The Lyapunov function $U$ in (33) is a generalization of the hybrid Lyapunov function in [1], which is constructed for NCS with non-switched protocols. Compared with [1], we introduce new functions $\theta_{1}$ and $\theta_{2}$ in (33) to accommodate the influence of the length of segments. It is interesting to note that when there is a single segment $\left(T_{1}=\infty\right)$, we can let the function $\theta_{1}$ satisfy $\theta_{1}(s) \equiv 1$ for all $s \geq 0$ (since $\theta_{1}$ is irrelevant in this case) and $U$ becomes $U(\xi)=V(x)+\gamma_{1} \phi_{1}\left(\tau_{1}\right) W_{1}^{2}(e, \kappa)$ as in [1]. In this case, we recover the MATI bounds in [1] by taking $M=1$ and $k_{1}=\gamma_{1}$, which is possible since we can choose parameters $\gamma_{2}, \pi_{1}, \pi_{2}, \rho_{2}$ in an arbitrary way as the segment $q=2$ will never be activated.

\section{Illustrative example}

In this section, we apply our results to stabilize the origin of a batch reactor in [14] over FlexRay. Consider the batch reactor plant of the form

$$
x_{p}=A_{p} x_{p}+B_{p} u \quad y=C_{p} x_{p}
$$

and the PI controller given by

$$
\dot{x}_{c}=A_{c} x_{c}+B_{c} y \quad u=C_{c} x_{c}+D_{c} y,
$$


Table 1

Comparison of MATIs for the batch reactor

\begin{tabular}{|l|c|c|}
\hline \hline & RR & TOD \\
\hline MATI for RR from [1] & 0.0093 & - \\
\hline MATI for TOD from [1] & - & 0.01084 \\
\hline $\begin{array}{l}\text { MATI for RR-TOD via (31) } \\
T_{1}=0.0186, T_{2}=0.01084\end{array}$ & 0.0062 & 0.01057 \\
\hline $\begin{array}{l}\text { MATI for RR-TOD via (31) } \\
T_{1}=0.5, T_{2}=1.5\end{array}$ & 0.0082 & 0.01079 \\
\hline
\end{tabular}

where $x_{p} \in \mathbb{R}^{4}, x_{c} \in \mathbb{R}^{2}, y \in \mathbb{R}^{2}$ and $u \in \mathbb{R}^{2}, A_{p}, B_{p}$, $C_{p}, A_{c}, B_{c}, C_{c}$ and $D_{c}$ are constant matrices that can be found in Section 4 of [14]. We consider the scenario where the controller is directly connected to the actuator and the sensor measurements are transmitted via a FlexRay network. In this case, we can still model the NCS as (22) although the output of the controller in (34) is of the form $u=g_{c}\left(x_{c}, y\right)$ instead of $u=g_{c}\left(x_{c}\right)$ (see Remark 1 in $[17])$.

We consider the scenario where RR is implemented during the static segments, and TOD during the dynamic segments. To estimate MATIs, one needs to verify Assumptions 5-7 to calculate (31) based on the obtained parameters. Let $x=\left(x_{p}, x_{c}\right)$ and $e=\hat{y}-y$. As shown in [1], Assumptions 5-6 hold with $H_{1}(x)=\left|\sqrt{2} A_{21} x\right|, H_{2}(x):=$ $\left|A_{21} x\right|, \rho_{1}=0.7071, L_{1}=15.73, \gamma_{1}=21.5275, \rho_{2}=$ $0.7071, L_{2}=15.73$ and $\gamma_{2}=15.9222$. Through calculations according to $(30)$, we obtain that $\delta_{1}=\delta_{2}=0$, $k_{1}=232.853, k_{2}=31.844$.

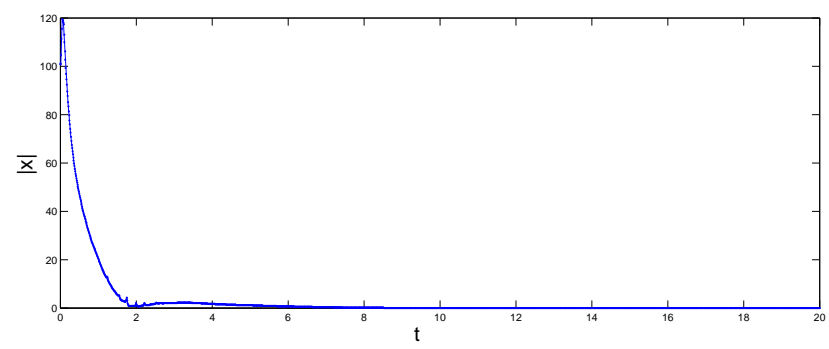

Fig. 5. Segments of length $T_{1}=0.0186$ and $T_{2}=0.01084$ $\tau_{s t}=0.0062$ and $\tau_{d y}=0.01057$ according to $(31)$

Given $T_{1}$ and $T_{2}>0$, we can estimate the MATI of the RR-TOD protocol using (31). Some numerical values are reported in Table 1 . We also provide the MATIs for non-switching protocols RR and TOD from [1] in Table 1. Compared with MATIs of non-switching protocols, the NCS over FlexRay requires more frequent transmissions to preserve stability of its closed-loop. This becomes evident when the lengths of the segments are small. For instance, consider the case when $T_{1}=0.0186$ and $T_{2}=0.01084$. Simulations show that $x$ does not convergence to zero when we use the MATI bounds for non-switched RR and TOD protocols given in Table 1.
This issue does not occur with the MATI bounds obtained with (31), see Figure 5.

\section{Conclusion}

We have shown how the emulation approach can be used to design controllers implemented over FlexRay for nonlinear systems. In contrast with the previous related works $[1,14,21,23]$, the protocol which governs transmissions in FlexRay switches in the sense that a different policy is used during the static and the dynamic segments. As a consequence, we needed to develop a novel hybrid model which describes NCS implemented on FlexRay under reasonable assumptions. We then proposed a hybrid Lyapunov function, which extends the one in [1], to NCS with switched protocols. We used this Lyapunov function to derive MATI bounds which depends on the lengths of the static and the dynamics segments, based on which we ensured the uniform global asymptotic stability property of a closed set. Note that the presented result is only an illustration of what is possible in terms of stabilization. We can employ the technique presented in the paper, i.e. the modeling technique and the Lyapunov function, to investigate the observer design problem for nonlinear systems whose measurements are sent over a network governed by FlexRay, see [24]. We may also take the present results as a starting point to consider other kinds of network-induced constraints such as quantization, time-delays, or packet drop-outs, by following similar lines as in $[6,13,20]$.

\section{References}

[1] D. Carnevale, A. R. Teel, and D. Nešić. A Lyapunov proof of an improved maximum allowable transfer interval for networked control systems. IEEE Transactions on Automatic Control, 52:892-897, 2007.

[2] G. Cena and A. Valenzano. On the properties of the flexible time division multiple access technique. IEEE Transactions on Industrial Informatics, 2:86-94, 2006.

[3] Flexray Consortium. FlexRay Communications System Protocol Specification V2.1 Rev.A, 2005.

[4] R. Goebel, R. G. Sanfelice, and A. R. Teel. Hybrid dynamical systems: Modeling, Stability, and Robustness. Princeton University Press, 2012.

[5] D. Goswami, R. Schneider, and S. Chakraborty. Reengineering cyber-physical control applications for hybrid communication protocols. In Proceedings of the Design, Automation $8 \mathcal{G}$ Test in Europe Conference $\& \mathcal{G}$ Exhibition, pages 2571-2576, 1-6, 2011.

[6] W.P.M.H. Heemels, A.R. Teel, N. van de Wouw, and D. Nešić. Networked control systems with communication constraints: Tradeoffs between transmission intervals, delays and performance. IEEE Transactions on Automatic Control, $55: 1781-1796,2010$.

[7] W.P.M.H. Heemels and N. van de Wouw. Stability and stabilization of networked control systems. In A. Bemporad, Maurice Heemels, and Mikael Johansson, editors, Networked Control System, volume 406 of Lecture notes in control 
and information sciences, pages 203-253. Springer-Verlag, London, 2010.

[8] Australia National Instruments. FlexRay Automotive Communication Bus Overview, 2009.

[9] A. Isidori. Nonlinear control systems. Springer-Verlag, 1995.

[10] A. Isidori. Nonlinear control systems: part II. SpringerVerlag, 1999.

[11] D. S. Laila and D. Nešić. Lyapunov based small-gain theorem for parameterized discrete-time interconnected ISS systems. In Proceedings of the 41th IEEE Conference on Decision and Control, pages 2292-2297, Las Vegas, USA, 2002.

[12] P. Naghshtabrizi and J. P. Hespanha. Analysis of distributed control systems with shared communication and computation resources. In Proceedings of the American Control Conference, pages 3384-3389, Saint Louis, USA, 2009 .

[13] D. Nešić and D. Liberzon. A unified framework for design and analysis of networked and quantized control systems. IEEE Transactions on Automatic Control, 54:732-747, 2009.

[14] D. Nešić and A. R. Teel. Input-output stability properties of networked control systems. IEEE Transactions on Automatic Control, 49:1650-1667, 2004.

[15] D. Nešić and A. R. Teel. Input-to-state stability of networked control systems. Automatica, 40:2121-2128, 2004.

[16] T. Pop, P. Pop, P. Eles, Z. Peng, and A. Andrei. Timing analysis of the Flexray communication protocol. Real-Time Systems, 39:205-235, 2008.

[17] R. Postoyan, N. van de Wounw, D. Nešić, and W.P.M.H. Heemels. Tracking control for nonlinear networked control systems. IEEE Transactions on Automatic Control, to appear.

[18] E. G. Schmidt and K. Schmidt. Message scheduling for the flexray protocol: the dynamic segment. IEEE Transactions on Vehicular Technology, 58:2160-2169, 2009.

[19] R. Shaw and B. Jackman. An introduction to flexray as an industrial network. In Proceedings of the IEEE International Symposium on Industrial Electronics, pages 1849-1854, Cambridge, UK, 2008.

[20] M. Tabbara and D. Nešić. Input-output stability of networked control systems with stochastic protocols and channels. IEEE Transactions on Automatic Control, 53:1160-1175, 2007.

[21] M. Tabbara, D. Nešić, and A. R. Teel. Networked control systems: emulation-based design. In F. Wang and D. Liu, editors, Networked control systems, pages 57-94. Springer, London, 2008.

[22] A. R. Teel and L. Praly. On assigning the derivative of a disturbance attenuation control Lyapunov function. Mathematics of Control, Signals, and Systems, 13:95-124, 2000.

[23] G. C. Walsh and H. Ye. Scheduling of networked control systems. IEEE Control Systems Magzine, 21:57-65, 2001.

[24] W. Wang, D. Nešić, and R. Postoyan. Design of observers implemented over flexray networks. In Proceedings of the Australian Control Conference, Canberra, Australia, 2014.

[25] W. Wang, D. Nešić, and R. Postoyan. Emulation-based stabilization of networked control systems implemented on flexray. In Proceedings of the IEEE Conference on Decision and Control, Los Angeles, USA, 2014.

\section{A Technical lemmas}

Lemma 3 Suppose that Assumption 7 holds. Then, the functions defined as

$\phi_{1}(t):=\left\{\begin{array}{ll}\bar{\phi}_{1}(t) & t \in\left[0, \tau_{\mathrm{MATI}}^{s}\right] \\ \rho_{1} & t \in\left[\tau_{\mathrm{MATI}}^{s}, \infty\right)\end{array} \phi_{2}(t):= \begin{cases}\bar{\phi}_{2}(t) & t \in\left[0, \tau_{\mathrm{MATI}}^{d}\right] \\ \rho_{2} & t \in\left[\tau_{\mathrm{MATI}}^{d}, \infty\right)\end{cases}\right.$

satisfy $\phi_{m}(t) \in\left[\rho_{\underline{m}}, 1 / \rho_{m}\right]$ for each $m \in\{1,2\}$ and all $t \in[0, \infty)$, where $\bar{\phi}_{m}$ is given by the solution to

$$
\dot{\bar{\phi}}_{m}=-2 L_{m} \bar{\phi}_{m}-\gamma_{m} \bar{\phi}_{m}^{2}-c_{m}\left(T_{m}\right) \quad \bar{\phi}_{m}(0)=\frac{1}{\rho_{m}}
$$

where $c_{m}(\cdot)$ come from (29) and $T_{m}>0$.

Proof. We first consider the solution $\bar{\phi}_{m}$ to (A.1) to determine the value of $\tau_{\text {MATI }, m}$ such that $\bar{\phi}_{m}\left(\tau_{\text {MATI }, m}\right)=\rho_{m}$. In view of (A.1),

$$
\begin{aligned}
& \tau_{\text {MATI }, m}=-\int_{1 / \rho_{m}}^{\rho_{m}} \frac{d \bar{\phi}_{m}}{\gamma_{m} \bar{\phi}_{m}^{2}+2 L_{m} \bar{\phi}_{m}+c_{m}\left(T_{m}\right)} \text { (A.2) } \\
& =-\frac{1}{\gamma_{m}} \int_{a_{m}}^{b_{m}} \frac{d z}{z^{2}-L_{m}^{2} / \gamma_{m}^{2}+c_{m}\left(T_{m}\right) / \gamma_{m}} \\
& =-\frac{1}{\gamma_{m}} \int_{a_{m}}^{b_{m}} \frac{d z}{z^{2}-\operatorname{sgn}\left(L_{m}-\sqrt{\gamma_{m} c_{m}\left(T_{m}\right)}\right)\left(L_{m} r_{m} / \gamma_{m}\right)^{2}}
\end{aligned}
$$

where $z=\bar{\phi}_{m}+L_{m} / \gamma_{m}, a_{m}:=1 / \rho_{m}+L_{m} / \gamma_{m}$, $b_{m}:=\rho_{m}+L_{m} / \gamma_{m}, r_{m}=\sqrt{\left|\frac{\gamma_{m} c_{m}\left(T_{m}\right)}{L_{m}^{2}}-1\right|}$ and $\operatorname{sgn}(\cdot)$ is the $\operatorname{sign}$ function with $\operatorname{sgn}(0)=0$. When $\sqrt{\gamma_{m} c_{m}\left(T_{m}\right)}>L_{m}$, (A.2) implies that $\tau_{\mathrm{MATI}, m}=$ $-\frac{1}{\gamma_{m}} \int_{a_{m}}^{b_{m}} \frac{d z}{z^{2}+\left(L_{m} r_{m} / \gamma_{m}\right)^{2}}=-\frac{1}{L_{m} r_{m}}\left[\arctan \left(\frac{b_{m} \gamma_{m}}{L_{m} r_{m}}\right)-\right.$ $\left.\arctan \left(\frac{a_{m} \gamma_{m}}{L_{m} r_{m}}\right)\right]$. In this case, we have that $\tau_{\text {MATI }, m}$ agrees with (31) noting that $\arctan \left(k_{2}\right)-\arctan \left(k_{1}\right)=$ $\arctan \left(\frac{k_{2}-k_{1}}{1+k_{1} k_{2}}\right)$ for all $k_{1}, k_{2} \geq 0$. When $\sqrt{\gamma_{m} c_{m}\left(T_{m}\right)}=$ $L_{m}$, (31) follows from $-\frac{1}{\gamma_{m}} \int_{a_{m}}^{b_{m}} \frac{d z}{z^{2}}=\frac{1}{\gamma_{m}}\left[\frac{1}{b_{m}}-\frac{1}{a_{m}}\right]$. When $\sqrt{\gamma_{m} c_{m}\left(T_{m}\right)}<L_{m}$, in view of (A.2),

$$
\begin{aligned}
& \tau_{\text {MATI }, m}=\frac{1}{\gamma_{m}} \int_{a_{m}}^{b_{m}} \frac{d z}{\left(L_{m} r_{m} / \gamma_{m}\right)^{2}-z^{2}} \\
& =\frac{1}{L_{m} r_{m}}\left[\operatorname{arctanh}\left(\frac{b_{m} \gamma_{m}}{L_{m} r_{m}}\right)-\operatorname{arctanh}\left(\frac{a_{m} \gamma_{m}}{L_{m} r_{m}}\right)\right]
\end{aligned}
$$

and (31) holds since $\operatorname{arctanh}\left(k_{2}\right)-\operatorname{arctanh}\left(k_{1}\right)=$ $\operatorname{arctanh}\left(\frac{k_{2}-k_{1}}{1-k_{1} k_{2}}\right)$. We then conclude the lemma by noting that $\phi_{m}(s)=\rho_{m}$ when $s \geq \tau_{\mathrm{MATI}, m}$. 
Let $\theta_{m}$ for $m \in\{1,2\}$ be the solution to

$$
\dot{\theta}_{m}(s)=\frac{1}{\phi_{m}(s)}\left(-\gamma_{m}+\left(c_{m}\left(T_{m}\right)-\delta_{m}\right) \theta_{m}(s)\right)
$$

with $\phi_{m}: \mathbb{R}_{\geq 0} \rightarrow \mathbb{R}_{>0}$ being defined in Lemma 3 and $\theta_{m}(0)=\Gamma_{m}\left(\bar{T}_{m}\right)$, where

$$
\Gamma_{m}\left(T_{m}\right):=\frac{\gamma_{m}}{c_{m}\left(T_{m}\right)-\delta_{m}}+\nu_{m}\left(T_{m}\right),
$$

$c_{m}$ and $\delta_{m}$ respectively come from (29) and (30). The function $\nu_{m}: \mathbb{R}_{\geq 0} \rightarrow \mathbb{R}_{\geq 0}$ is to be determined using the lemma below.

Lemma 4 For any $T_{m}>0$ and $m \in\{1,2\}$, there exists a continuous function $\nu_{m}: \mathbb{R}_{\geq 0} \rightarrow \mathbb{R}_{\geq 0}$ such that the solution $\theta_{m}$ to (A.3) satisfies the properties listed below.

(1) $\theta_{m}\left(T_{m}\right)=M$ and $\theta_{m}(s)$ is non-decreasing on $[0, M]$ when $s$ increases, where $s \in\left[0, T_{m}\right]$ and $M=\max \left\{1, \frac{\gamma_{2} \rho_{1} \rho_{2}}{\gamma_{1} \pi_{2}^{2}}, \frac{\gamma_{1} \rho_{1} \rho_{2}}{\gamma_{2} \pi_{1}^{2}}\right\}$.

(2) $\theta_{m}(0) \leq \frac{\gamma_{3-m} \rho_{1} \rho_{2}}{\gamma_{m} \pi_{3-m}^{2}} \theta_{3-m}\left(T_{3-m}\right)$.

Proof. To determine the function $\nu_{m}$, we study the following linear differential equations

$$
\begin{aligned}
& \dot{\bar{\theta}}_{m}=\rho_{m}\left(-\gamma_{m}+\left(c_{m}\left(T_{m}\right)-\delta_{m}\right) \bar{\theta}_{m}\right) \\
& \dot{\theta}_{m}=\frac{1}{\rho_{m}}\left(-\gamma_{m}+\left(c_{m}\left(T_{m}\right)-\delta_{m}\right) \underline{\theta}_{m}\right)
\end{aligned}
$$

with initial conditions $\bar{\theta}_{m}(0)=\frac{\gamma_{m}}{c_{m}\left(T_{m}\right)-\delta_{m}}+\bar{\nu}_{m}$ and $\underline{\theta}_{m}(0)=\frac{\gamma_{m}}{c_{m}\left(T_{m}\right)-\delta_{m}}+\underline{\nu}_{m}$ for some $\bar{\nu}_{m}, \underline{\nu}_{m}>0$. The solutions to (A.5) satisfy for $s \geq 0$

$$
\begin{aligned}
& \bar{\theta}_{m}(s)=\frac{\gamma_{m}}{c_{m}\left(T_{m}\right)-\delta_{m}}+\bar{\nu}_{m} \exp \left(\left(c_{m}\left(T_{m}\right)-\delta_{m}\right) \rho_{m} s\right) \\
& \underline{\theta}_{m}(s)=\frac{\gamma_{m}}{c_{m}\left(T_{m}\right)-\delta_{m}}+\underline{\nu}_{m} \exp \left(\frac{c_{m}\left(T_{m}\right)-\delta_{m}}{\rho_{m}} s\right) .
\end{aligned}
$$

We select $\bar{\nu}_{m}, \underline{\nu}_{m}$ such that $\bar{\theta}_{m}\left(T_{m}\right)=M, \underline{\theta}_{m}\left(T_{m}\right)=M$, i.e.

$$
\begin{aligned}
& \bar{\nu}_{m}\left(T_{m}\right)=\frac{\left(c_{m}\left(T_{m}\right)-\delta_{m}\right) M-\gamma_{m}}{\left(c_{m}\left(T_{m}\right)-\delta_{m}\right) \exp \left(\left(c_{m}\left(T_{m}\right)-\delta_{m}\right) \rho_{m} T_{m}\right)} \\
& \underline{\nu}_{m}\left(T_{m}\right)=\frac{\left(c_{m}\left(T_{m}\right)-\delta_{m}\right) M-\gamma_{m}}{\left(c_{m}\left(T_{m}\right)-\delta_{m}\right) \exp \left(\frac{c_{m}\left(T_{m}\right)-\delta_{m}}{\rho_{m}} T_{m}\right)},
\end{aligned}
$$

where $\delta_{m} \geq 0$ and $M \geq 1$ are constants. Note that $\bar{\nu}_{m}, \underline{\nu}_{m}$ in (A.7) satisfies $\bar{\nu}_{m}, \underline{\nu}_{m} \geq 0$ since $M \geq 1$ and $c_{m}\left(T_{m}\right) \geq \gamma_{m}+\delta_{m}$ for any $T_{m}>0$ (see footnote 4 for explanations) and $k_{m} \geq \gamma_{m}$ from its definition. Consequently, the solutions $\bar{\theta}_{m}, \underline{\theta}_{m}$ to (A.5) are nondecreasing. We next show $\bar{\theta}_{m}(0), \underline{\theta}_{m}(0) \in[0, M]$ to conclude that $\bar{\theta}_{m}(s), \underline{\theta}_{m}(s) \in[0, M]$ for all $s \in\left[0, T_{m}\right]$.

From the definition of $d_{m}(s)$ in (30), it is straightforward that $d_{m}(s)<\frac{\gamma_{m}}{k_{m}}$ for each $s>0$ and $M \geq \frac{2 \gamma_{m}}{k_{m}}$. We can also get that $d_{m}(s)<\frac{k_{m} M-\gamma_{m}}{k_{m}} \leq \frac{\gamma_{m}}{k_{m}}$ for all $s>0$ when $M \leq \frac{2 \gamma_{m}}{k_{m}}$. Recalling the definition of $c_{m}$ in (29), the fact that $k_{m} \geq \frac{\gamma_{m}^{2} \pi_{3-m}^{2}}{\gamma_{3-m} \rho_{1} \rho_{2}}, d_{m}(s)<\frac{\gamma_{m}}{k_{m}}$ for all $s>0$ and $M \geq 1$, it follows that

$$
\begin{aligned}
\bar{\theta}_{m}(0) & =\frac{\gamma_{m}}{\frac{\gamma_{m}}{\gamma_{m} / k_{m}-d_{m}\left(T_{m}\right)}+\frac{1}{\rho_{m} T_{m}}}+\bar{\nu}_{m}\left(T_{m}\right) \\
& \leq \frac{\gamma_{3-m} \rho_{1} \rho_{2}}{\gamma_{m} \pi_{3-m}^{2}}-d_{m}\left(T_{m}\right)+\bar{\nu}_{m}\left(T_{m}\right) .
\end{aligned}
$$

We then derive that $\bar{\theta}_{m}(0) \leq \frac{\gamma_{3-m} \rho_{1} \rho_{2}}{\gamma_{m} \pi_{3-m}^{2}} \leq M$ when $\bar{\nu}_{m}(s) \leq d_{m}(s)$ holds for any $s>0$, which we now prove.

Case I: $M \leq \frac{2 \gamma_{m}}{k_{m}}$. Note that we can rewrite functions $\bar{\nu}_{m}$ and $d_{m}$ respectively as $f_{m}\left(x_{m}\right)$ and $f_{m}\left(y_{m}\right)$, where $x_{m}:=c_{m}\left(T_{m}\right)-\delta_{m}, y_{m}:=k_{m}$ and

$$
f_{m}(x):=\frac{x M-\gamma_{m}}{x \exp \left(x \rho_{m} T_{m}\right)} .
$$

Since $c_{m}\left(T_{m}\right)-\delta_{m} \geq k_{m}$ for all $T_{m}>0$ as discussed in footnote $4, \bar{\nu}_{m}(s) \leq \bar{d}_{m}(s)$ holds for any $s>0$ if $f_{m}(\cdot)$ is non-increasing, which is true. Indeed, $\frac{\partial f_{m}\left(x_{m}\right)}{\partial x_{m}}<0$ since $M \geq 1$ and $x_{m}=c_{m}\left(T_{m}\right)-\delta_{m} \geq \gamma_{m}+\frac{1}{\rho_{m} T_{m}}$ for all $T_{m}>0$, where

$$
\begin{aligned}
\frac{\partial f_{m}}{\partial x_{m}}= & \frac{x_{m} M-\left(1+x_{m} \rho_{m} T_{m}\right)\left(x_{m} M-\gamma_{m}\right)}{x_{m}^{2} \exp \left(x_{m} \rho_{m} T_{m}\right)} \\
= & \frac{\gamma_{m}+\rho_{m} T_{m} \frac{\gamma_{m}^{2}}{4}-\rho_{m} T_{m}\left(x_{m}-\frac{\gamma_{m}}{2}\right)^{2}}{x_{m}^{2} \exp \left(x_{m} \rho_{m} T_{m}\right)} \\
& +\frac{\rho_{m} T_{m} x_{m}^{2}(1-M)}{x_{m}^{2} \exp \left(x_{m} \rho_{m} T_{m}\right)} .
\end{aligned}
$$

Case II: $M \geq \frac{2 \gamma_{m}}{k_{m}}$. Note that we can rewrite (30) and obtain

$$
d(s)=\frac{\bar{k}_{m} M-\gamma_{m}}{\bar{k}_{m} \exp \left(\bar{k}_{m} \rho_{m} s\right)} \text { when } M \geq \frac{2 \gamma_{m}}{k_{m}},
$$

with letting $\bar{k}_{m}:=\frac{k_{m} \gamma_{m}}{k_{m} M-\gamma_{m}}$. Note that $\bar{k}_{m}=$ $\frac{k_{m} \gamma_{m}}{k_{m} M-\gamma_{m}} \leq k_{m}$ in this case and consequently $c_{m}(s)-$ $\delta_{m} \geq \bar{k}_{m}$ for all $s>0$ when $M \geq \frac{2 \gamma_{m}}{k_{m}}$. Applying the same technique as in Case I, we can conclude that $\bar{\nu}_{m}(s) \leq d_{m}(s)$ holds for any $s>0$ and $M \geq \frac{2 \gamma_{m}}{k_{m}}$, and hence it holds for all $M \geq 1$.

By now, we have that $\bar{\theta}_{m}(0) \in[0, M]$, also $\underline{\theta}_{m}(0) \in$ $[0, M]$ since $0 \leq \underline{\nu}_{m}(s) \leq \bar{\nu}_{m}(s)$ for all $s \geq 0$. In view of Lemma 3 that $\phi_{m}(s) \in\left[\rho_{m}, 1 / \rho_{m}\right]$ for all $s \geq 0$, we therefore respectively upper-bound and lower-bound $\theta_{m}$, the solution to (A.3), by $\bar{\theta}_{m}$ and $\underline{\theta}_{m}$ in (A.6) and by selecting $\nu_{m}$ such that $\underline{\nu}_{m}(s) \leq \nu_{m}(s) \leq \bar{\nu}_{m}(s)$ for all $s \geq 0$. 
As $\underline{\theta}_{m}(s)$ and $\bar{\theta}_{m}(s) \in[0, M]$, there exists a function $\nu_{m}$ satisfying the above condition such that $\theta_{m}\left(T_{m}\right)=M$ and $\theta_{m}(s) \in[0, M]$ for all $s \in\left[0, T_{m}\right]$. Then, we can derive from $\theta_{m}(0) \leq \bar{\theta}_{m}(0) \leq \frac{\gamma_{3-m} \rho_{m} \rho_{3-m}}{\gamma_{m} \pi_{3-m}^{2}}$ that $\theta_{m}(0) \leq$ $\frac{\gamma_{3-m} \rho_{m} \rho_{3-m}}{\gamma_{m} \pi_{3-m}^{2}} \theta_{3-m}\left(T_{m}\right)$ since $\theta_{1}\left(T_{1}\right)=\theta_{2}\left(T_{2}\right)=M$ and $M>1$.

\section{B Proof of the main results}

Proof of Proposition 2. Let $T_{1}, T_{2}>0$ be given. Let $M:=\max \left\{1, \frac{\gamma_{2} \rho_{1} \rho_{2}}{\gamma_{1} \pi_{2}^{2}}, \frac{\gamma_{1} \rho_{1} \rho_{2}}{\gamma_{2} \pi_{1}^{2}}\right\}, c_{1}, c_{2}: \mathbb{R}_{>0} \rightarrow \mathbb{R}$ be defined as (29). We consider the function $U$ defined in (33) with the functions $\theta_{1}$ and $\theta_{2}$ defined by the solution to the differential equations (A.3). The functions $\phi_{1}$ and $\phi_{2}$ are defined as $\phi_{1}(s)=\vec{\phi}_{1}(s)$ for $s \in\left[0, \tau_{\text {MATI }}^{s}\right]$ and $\phi_{1}(s)=\rho_{1}$ for $s \in\left[\tau_{\text {MATI }}^{s}, \infty\right), \phi_{2}(s)=\bar{\phi}_{2}(s)$ for $s \in\left[0, \tau_{\text {MATI }}^{d}\right]$ and $\phi_{2}(s)=\rho_{2}$ for $s \in\left[\tau_{\text {MATI }}^{d}, \infty\right)$, where $\bar{\phi}_{1}$ and $\bar{\phi}_{2}$ are solutions to (A.1). We note that $U$ is continuous and locally Lipschitz in $x, e, \tau_{1}, \tau_{2}$ in view of (A.1), (A.3), Assumption 5 and the continuity of $\mathcal{F}$ and $\mathcal{G}$. We now show that item (2) of Proposition 2 holds. Let $\xi \in C \cup D$ with $q=1$. In view of Lemmas 3 and 4 in Appendix A,

$$
\begin{array}{ll}
\bar{\phi}_{1}\left(\tau_{1}\right) \in\left[\rho_{1}, 1 / \rho_{1}\right] & \forall \tau_{1} \in\left[0, \tau_{\mathrm{MATI}}^{s}\right] \\
\theta_{1}\left(\tau_{2}\right) \in\left[\Gamma\left(T_{1}\right), M\right] & \forall \tau_{2} \in\left[0, T_{1}\right],
\end{array}
$$

where the function $\Gamma_{1}$ come from (A.4). Note that $\Gamma_{1}(s) \geq \frac{\gamma_{1}}{c_{1}(s)-\delta_{1}}+\underline{\nu}_{1}(s)$ for all $s>0$ as showed in the proof of Lemma 4 . The two terms of the righthand side of the inequality are all strict positive functions (see footnote 4 and $(\mathrm{A} .7))$. The function $\frac{\gamma_{1}}{c_{1}(s)-\delta_{1}}$ is nondecreasing when its argument grows, approaches to 0 when $T_{1} \rightarrow 0$ and $\frac{\gamma_{1}}{k_{1}}$ when $T_{1} \rightarrow \infty$. Moreover, the function $\underline{\nu}_{1}$ is non-increasing, $\underline{\nu}_{1}$ approaches $M$ when $T_{1} \rightarrow 0$ and $\underline{\nu}_{1}(\infty)=0$. Thus, with noting that $M \geq \frac{\gamma_{1}}{k_{1}}$ since $k_{1} \geq \gamma_{1}$ and $M \geq 1$ from the definition of $M$ and $k_{1}$, we have that $\Gamma_{1}(s) \geq \frac{\gamma_{1}}{k_{1}}$ for all $s>0$. Then, in view of Assumptions 5-6,

$$
\underline{\alpha}_{U}(|(x, e)|) \leq U(\xi) \leq \bar{\alpha}_{U}(|(x, e)|),
$$

where $\underline{\alpha}_{U}: s \mapsto \min \left\{\underline{\alpha}_{V}\left(\frac{s}{2}\right), \frac{\gamma_{1}^{2} \rho_{1}}{2 k_{1}} \underline{\alpha}_{W}^{2} s\right\} \in \mathcal{K}_{\infty}$ and $\bar{\alpha}_{U}(s): s \mapsto \max \left\{2 \bar{\alpha}_{V}(s), 2 \frac{\gamma_{1} M}{\rho_{1}} \bar{\alpha}_{W}^{2} s\right\} \in \mathcal{K}_{\infty}$. The constructions of $\bar{\alpha}_{U}$ and $\underline{\alpha}_{U}$ use Remark 2.3 in [11], where it is shown that $\min \left\{\alpha_{1}\left(\frac{s_{1}+s_{2}}{2}\right), \alpha_{2}\left(\frac{s_{1}+s_{2}}{2}\right)\right\} \leq$ $\alpha_{1}\left(s_{1}\right)+\alpha_{2}\left(s_{2}\right) \leq \max \left\{2 \alpha_{1}\left(s_{1}+s_{2}\right), 2 \alpha_{2}\left(s_{1}+s_{2}\right)\right\}$ for any $\alpha_{1}, \alpha_{2} \in \mathcal{K}_{\infty}$ and $s_{1}, s_{2} \geq 0$. By using similar arguments for the case where $q=2$, we deduce that item 2) holds.

We now focus on item (3) of Proposition 2. We omit below the dependencies of $\phi_{1}$ and $\theta_{1}$ respectively on $\tau_{1}$ and $\tau_{2}$ for the sake of convenience. In view of pages 99-100 in [22] and Assumption 6, and item (iii) of Assumption 5 , for all $\xi \in C$ with $q=1$,

$$
\begin{aligned}
U^{\circ} & (\xi ; \mathcal{F}(\xi)) \\
\leq & -\varrho_{1}(|x|)-\varrho_{1}(|e|)-H_{1}^{2}(x)+\gamma_{1}^{2} W_{1}^{2}(e, \kappa) \\
& +\gamma_{1} \dot{\phi}_{1} \theta_{1} W_{1}^{2}(e, \kappa)+\gamma_{1} \phi_{1} \dot{\theta}_{1} W_{1}^{2}(e, \kappa) \\
& +2 \gamma_{1} \phi_{1} \theta_{1} W_{1}(e, \kappa)\left(L_{1} W_{1}(e, \kappa)+H_{1}(x)\right) \\
= & -\varrho_{1}(|x|)-\varrho_{1}(|e|)-H_{1}^{2}(x) \\
& +2 \gamma_{1} \phi_{1} \theta_{1} W_{1}(e, \kappa) H_{1}(x)-\gamma_{1}^{2} \phi_{1}^{2} \theta_{1} W_{1}^{2}(e, \kappa) \\
& +\gamma_{1}\left(\dot{\phi}_{1}+2 L_{1} \phi_{1}+\gamma_{1} \phi_{1}^{2}+c_{1}\left(T_{1}\right)\right) \theta_{1} W_{1}^{2}(e, \kappa) \\
& +\gamma_{1}\left(\phi_{1} \dot{\theta}_{1}+\gamma_{1}-c_{1}\left(T_{1}\right) \theta_{1}\right) W_{1}^{2}(e, \kappa) .
\end{aligned}
$$

Hence, in view of Lemmas 3-4 and the fact that $\gamma_{1} \delta_{1} \theta_{1} W_{1}^{2}(e, \kappa) \geq(M-1) \gamma_{1}^{2} \phi_{1}^{2} \theta_{1} W_{1}^{2}(e, \kappa)$ as $\delta_{1}=$ $\frac{(M-1) \gamma_{1}}{\rho_{1}^{2}}$ from (30), we derive that

$$
\begin{aligned}
U^{\circ} & (\xi ; \mathcal{F}(\xi)) \\
\leq & -\varrho_{1}(|x|)-\varrho_{1}(|e|)-\left(H_{1}(x)-\gamma_{1} \phi_{1} \theta_{1} W_{1}(e, \kappa)\right)^{2} \\
& +\gamma_{1}^{2} \phi_{1}^{2} \theta_{1}^{2} W_{1}^{2}(e, \kappa)-M \gamma_{1}^{2} \phi_{1}^{2} \theta_{1} W_{1}^{2}(e, \kappa) \\
& +\gamma_{1}\left(\dot{\phi}_{1}+2 L_{1} \phi_{1}+\gamma_{1} \phi_{1}^{2}+c_{1}\left(T_{1}\right)\right) \theta_{1} W_{1}^{2}(e, \kappa) \\
& +\gamma_{1}\left(\phi_{1} \dot{\theta}_{1}+\gamma_{1}-\left(c_{1}\left(T_{1}\right)-\delta_{1}\right) \theta_{1}\right) W_{1}^{2}(e, \kappa) \\
\leq & -\varrho_{1}(|x|)-\varrho_{1}(|e|)-\gamma_{1}^{2} \phi_{1}^{2} \theta_{1} W_{1}^{2}(e, \kappa)\left(M-\theta_{1}\right) \\
\leq & -\varrho_{1}(|x|)-\varrho_{1}(|e|) .
\end{aligned}
$$

Noting the fact that $\varrho_{1}$ is positive definite there exists a continuous, positive definite function $\varrho$ such that

$$
U^{\circ}(\xi ; \mathcal{F}(\xi)) \leq-\varrho\left(|\xi|_{\mathcal{A}}\right)
$$

We can derive a similar conclusion as in (B.4) for the case when $q=2$ by following the same procedure. Hence, item 3) of Proposition 2 holds.

Let $\xi \in D$. We only present the analysis for the case when $q=1$ as the proof follows the same lines when $q=2$.

Case I: transmission jump. Let $\xi \in \mathbb{R}^{n_{x}} \times \mathbb{R}^{n_{e}} \times \mathbb{Z}_{>0} \times$ $D_{1}^{\text {trans }}$ (recall that the set $D_{1}^{\text {trans }}$ is defined in (15)). In view of (27), using that $\phi_{1}(0)=1 / \rho_{1},($ B.1) and item 2) of Assumption 5, we obtain

$$
\begin{aligned}
U & (\mathcal{G}(\xi)) \\
& =V(x)+\gamma_{1} \phi_{1}(0) \theta_{1}\left(\tau_{2}\right) W_{1}^{2}(h(\kappa, e, 1), \kappa+1) \\
& \leq V(x)+\gamma_{1} \frac{1}{\rho_{1}} \rho_{1}^{2} \theta_{1}\left(\tau_{2}\right) W_{1}^{2}(e, \kappa) \\
& \leq V(x)+\gamma_{1} \phi_{1}\left(\tau_{1}\right) \theta_{1}\left(\tau_{2}\right) W_{1}^{2}(e, \kappa)=U(\xi) .
\end{aligned}
$$


Case II: segment switch. Let $\xi \in \mathbb{R}^{n_{x}} \times \mathbb{R}^{n_{e}} \times \mathbb{Z}_{\geq 0} \times D_{1}^{\mathrm{seg}}$. In view of (27) and Lemma 3,

$$
\begin{aligned}
U(\mathcal{G}(\xi)) & =V(x)+\gamma_{2} \phi_{2}\left(\tau_{1}\right) \theta_{2}(0) W_{2}^{2}(e, \kappa) \\
& \leq V(x)+\gamma_{2} \frac{1}{\rho_{2}} \theta_{2}(0) W_{2}^{2}(e, \kappa) .
\end{aligned}
$$

On the other hand, we deduce from Assumption 5 that $W_{2}(e, \kappa) \leq \pi_{1} W_{1}(e, \kappa)$ with $\pi_{1}=\bar{\alpha}_{W_{2}} / \underline{\alpha}_{W_{1}}$. Consequently, with the fact that $\tau_{2}=T_{1}$ and $\theta_{2}(0) \leq$ $\frac{\gamma_{1} \rho_{1} \rho_{2}}{\gamma_{2} \pi_{1}^{2}} \theta_{1}\left(T_{1}\right)$ according to Lemmas $3-4$, it follows that

$$
\begin{aligned}
U(\mathcal{G}(\xi)) & \leq V(x)+\gamma_{2} \frac{1}{\rho_{2}} \theta_{2}(0) \pi_{1}^{2} W_{1}^{2}(e, \kappa) \\
& \leq V(x)+\gamma_{1} \phi_{1}\left(\tau_{1}\right) \theta_{1}\left(\tau_{2}\right) W_{1}^{2}(e, \kappa)=U(\xi) .
\end{aligned}
$$

This completes the proof noting that $D=\mathbb{R}^{n_{x}} \times \mathbb{R}^{n_{e}} \times$ $\mathbb{Z}_{\geq 0} \times\left(D_{1} \cup D_{2}\right)$ and $D_{1}=D_{1}^{\text {trans }} \cup D_{1}^{\text {seg }}$.

Proof of Theorem 1. We use similar arguments as in Proposition 3.27 in [4] to show that the closed set $\mathcal{A}$ is UGpAS for system (23) (the only difference comes from the fact that the Lyapunov function $U$ is not continuously differentiable but locally Lipschitz in $\left.x, e, \tau_{1}, \tau_{2}\right)$.

We note that (3.7a), (3.7b) and (3.9) of Proposition 3.27 in [4] are verified in view of Proposition 2. We next show the following persistent flow condition holds: for any $r>0$, there exists $\bar{\gamma} \in \mathcal{K}_{\infty}$ and $N \geq 0$ such that for every solution $\xi$ to system (23) with $|\xi(0,0)|_{\mathcal{A}} \in(0, r]$, $(t, j) \in \operatorname{dom} \xi, t+j \geq K$ for any $K>0$ imply $t \geq$ $\bar{\gamma}(K)-N$. Consider any $r, K>0$ and a solution $\xi$ to (23) with $|\xi(0,0)|_{\mathcal{A}} \in(0, r]$. Recall that $\varepsilon$ in (23) is the length of a minislot and it is the lower bound of the intertransmission time for dynamic segments. Furthermore, note that $\varepsilon$ is far less than the inter-transmission time interval $\tau_{\text {MATI }}^{s}$ for static segments, see Section 4.3. Thus, in view of the periodical segment switching behavior of system (23) and noting that there are at most two segment jumps in one communication cycle, we have that

$$
t \geq \varepsilon\left(j-1-2\left\lceil\frac{t}{T_{1}+T_{2}}\right\rceil\right) .
$$

Then, $t\left(1+2 \varepsilon\left(\frac{t}{T_{1}+T_{2}}+1\right)\right)+\varepsilon \geq \varepsilon j$ and then $t+j \geq$ $K$ implies that $M t+1 \geq K$ with $M:=\left(\frac{1}{\varepsilon}+\frac{2}{T_{1}+T_{2}}+3\right)$. Consequently, we derive that

$$
t \geq \bar{\gamma}(K)-N
$$

with $\bar{\gamma}(s)=\frac{s}{M}$ and $N=\frac{1}{M}$. Then, by using similar lines as in the proof of Proposition 3.27 in [4] and the results on pages 99-100 in [22], we obtain that (2) holds. 